\title{
Solar Torque Management for the Near Earth Asteroid Scout CubeSat Using Center of Mass Position Control
}

\author{
Juan Orphee ${ }^{1}$ and Andrew Heaton. ${ }^{2}$ \\ NASA MSFC, Huntsville, AL, 35812 \\ Ben Diedrich ${ }^{3}$ and Brandon C. Stiltner ${ }^{4}$ \\ Jacobs ESSSA Group / NASA MSFC, Huntsville, AL, 35812
}

\begin{abstract}
A novel mechanism, the Active Mass Translator (AMT), has been developed for the NASA Near Earth Asteroid (NEA) Scout mission to autonomously manage the spacecraft momentum. The NEA Scout CubeSat will launch as a secondary payload onboard Exploration Mission 1 of the Space Launch System. To accomplish its mission, the CubeSat will be propelled by an 86 square-meter solar sail during its two-year journey to reach asteroid 1991VG. NEA Scout's primary attitude control system uses reaction wheels for holding attitude and performing slew maneuvers, while a cold gas reaction control system performs the initial detumble and early trajectory correction maneuvers. The AMT control system requirements, feedback architecture, and control performance will be presented. The AMT reduces the amount of reaction control propellant needed for momentum management and allows for smaller capacity reaction wheels suitable for the limited $6 \mathrm{U}$ spacecraft volume. The reduced spacecraft mass allows higher in-space solar sail acceleration, thus reducing time-offlight. The reduced time-of-flight opens the range of possible missions, which is limited by the lifetime of typical non-radiation tolerant CubeSat avionics exposed to the deep-space environment.
\end{abstract}

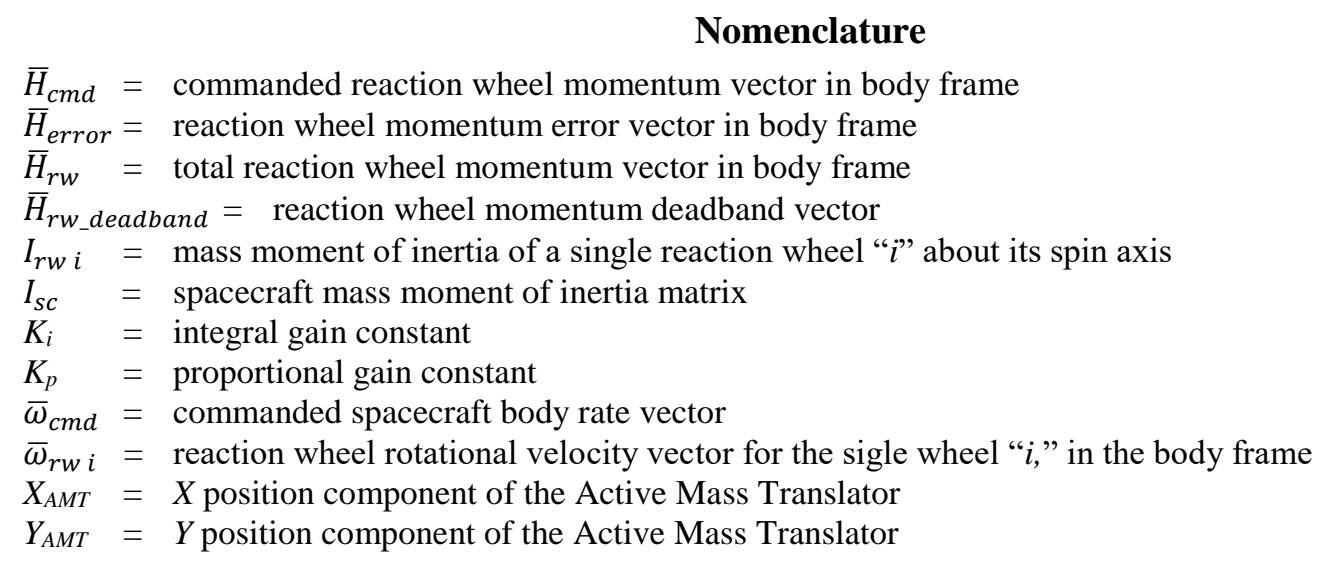

\section{Introduction}

The Near Earth Asteroid (NEA) Scout spacecraft is a 6U CubeSat that is currently under development as a joint effort between NASA Marshall Space Flight Center (MSFC) and the Jet Propulsion Laboratory (JPL). NEA Scout will launch on Space Launch System (SLS) Exploration Mission 1 (EM-1), currently scheduled to launch in 2019. NEA Scout's primary mission objective is to rendezvous with a near Earth asteroid 1991VG [1] and perform science imagery. The scientific objectives are to better characterize the target asteroid's orbit, and to take images of the

${ }^{1}$ Aerospace Engineer, NASA MSFC, Huntsville AL 35812, and AIAA Member.

${ }^{2}$ Senior Aerospace Engineer, NASA MSFC, Huntsville AL 35812, and AIAA Member.

${ }^{3}$ Aerospace Engineer, Jacobs ESSSA Group / NASA MSFC, Huntsville AL 35812.

${ }^{4}$ Aerospace Engineer, Jacobs ESSSA Group / NASA MSFC, Huntsville AL 35812, and AIAA Senior Member. 
asteroid's surface. The NEA Scout spacecraft and mission have been described in more detail in several publications [Error! Reference source not found., 2, 4, Error! Reference source not found.]. This paper will describe the overall spacecraft architecture, and will focus on the Active Mass Translator (AMT) system and its control performance.

The paper is organized as follows: Section II provides an overview of the NEA Scout CubeSat. Section III contains an overview of primary actuators and control sensors, including an in-depth description of the AMT hardware. Section IV details the AMT working principle, its mission requirements, and control design. Section V demonstrates the AMT performance against the requirements listed in Section IV. Finally, conclusions are provided in Section VI.

\section{NEA Scout Overview}

NEA Scout is a $6 \mathrm{U}$ CubeSat with an $86 \mathrm{~m}^{2}$ solar-sail used for propulsion. An image of the NEA Scout CubeSat is shown below, Figure 1, with the solar sail in its stowed configuration and solar panels deployed. The Attitude Control System (ACS) hardware is composed of two primary control actuators: the Reaction Wheel (RW) control system, the Reaction Control System (RCS), and a momentum management actuator AMT. Also shown, are the attitude determination sensors: three sun sensors, an Inertial Measurement Unit (IMU), and a star tracker.

The functions of each control actuator and sensors are discussed in Section Error! Reference source not found.. An in-depth description and analytical performance assessment of NEA Scout's RCS is provided by Stiltner et al. [Error! Reference source not found.], and more details on precision science pointing using reaction wheel control can be found in Orphee et al. [7].

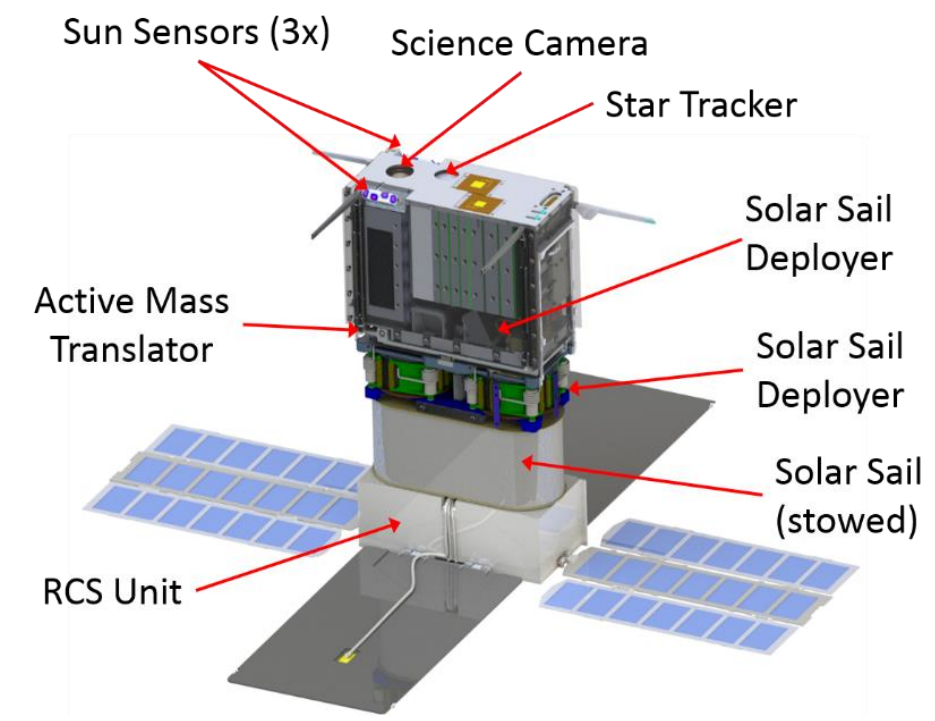

Figure 1. CAD images of NEA Scout showing the spacecraft in two different orientations and calling out the names and locations of various spacecraft components.

NEA Scout is divided into three major pieces as shown in Figure 2. Starting at the bottom of the figure, the solar panels are attached to the RCS unit. The RCS unit occupies approximately $2 \mathrm{U}$ of the spacecraft's volume and is outlined in green. Just above the RCS unit, is the solar sail and sail deployer. These items occupy the middle $2 \mathrm{U}$ of the spacecraft's volume and are outlined in blue. Last, the top portion, outlined in orange, is the spacecraft's avionics bus, and occupies the remaining $2 \mathrm{U}$ of volume. This last portion contains the science camera, the star tracker, flight computer, reaction wheel assembly, and other avionics components. For the majority of NEA Scout's mission, this side of the spacecraft will be pointed away from the sun and in the shadow of the solar sail. Figure 3 provides an image of NEA Scout before and after the solar sail is deployed. The AMT divides the avionics bus (orange) and sail plus sail deployer (blue) sections. 


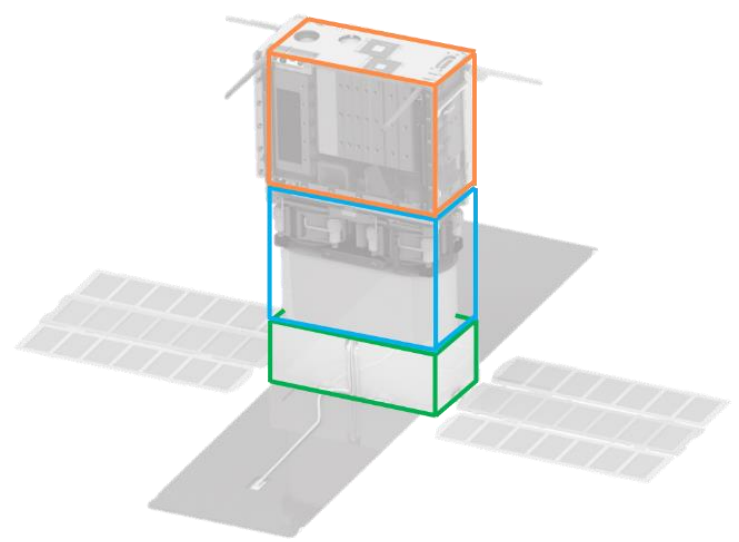

Figure 2. CAD image of NEA Scout calling out the three major pieces of the spacecraft: RCS unit (green), solar sail and deployer mechanism (blue), and avionics bus (orange).

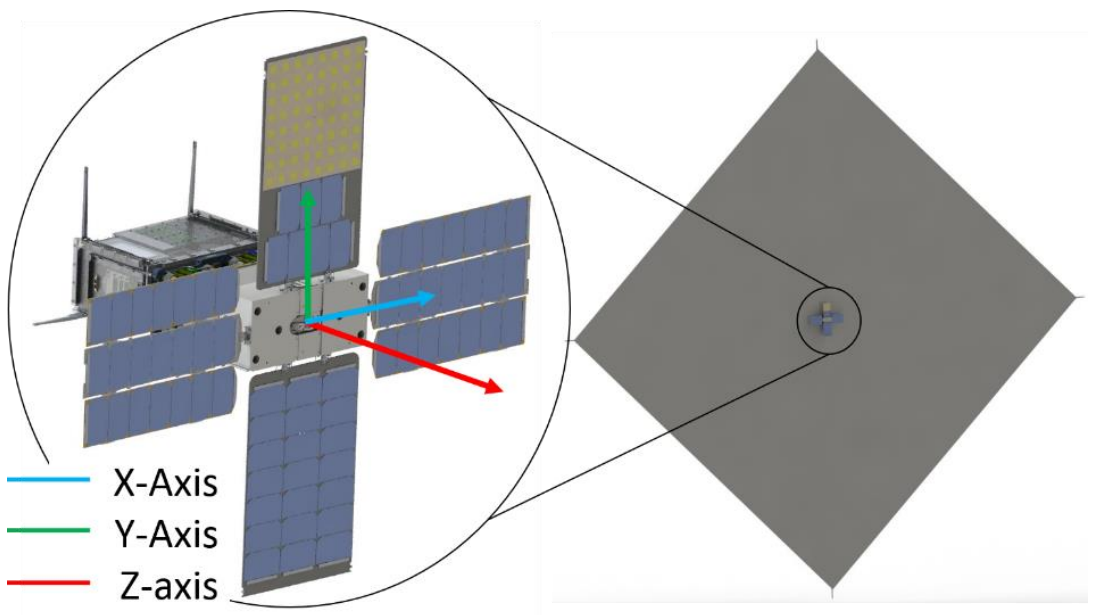

Figure 3. (left) Image of NEA Scout before solar sail deployment and (right) NEA Scout after solar sail deployment. The grey diamond on the right is the deployed solar sail, which is $86 \mathrm{~m}^{2}$ in size.

\section{NEA Scout ACS Overview}

\section{A. Actuators}

\section{Reaction Wheel System}

The reaction wheel hardware is composed of four reaction wheels with $0.015 \mathrm{~N}-\mathrm{m}$-s (Newton meter second) capacity each. The reaction wheels are arranged in pyramidal fashion with each spin axis approximately 60 degrees off the sail normal body axis. This arrangement allows for redundancy in case one of the reaction wheels fails. The RW control software uses a Moore-Penrose pseudo inverse allocation algorithm to distribute the commanded torque to each reaction wheel. In case of a single reaction wheel failure, a new allocation matrix can be uploaded from the ground.

\section{2. $R C S$}

The RCS unit is shown in Figure 1 and Figure 2. The unit occupies approximately $2 \mathrm{U}$ of volume on NEA Scout and contains $1.25 \mathrm{~kg}$ of propellant when full. The propellant is refrigerant R236fa . A conceptual image of the RCS unit is shown in Figure 4. The four circular features at the corners represent the attitude control jets, and arrows are used to depict the direction of thrust for each jet. The attitude control jets are oriented so that firing any pair generates a torque about one of the spacecraft body axes. The two circular features near the center are the axial jets, with thrust acting along the negative z-axis (into the page). The RCS is primarily used for detumble after ejection, an initial trajectory correction maneuver, momentum management for the non-sail deployed configuration, momentum 
management of Z-axis (sail normal) torques for sail deployed configuration, and any safing event requiring either momentum management or attitude recovery.

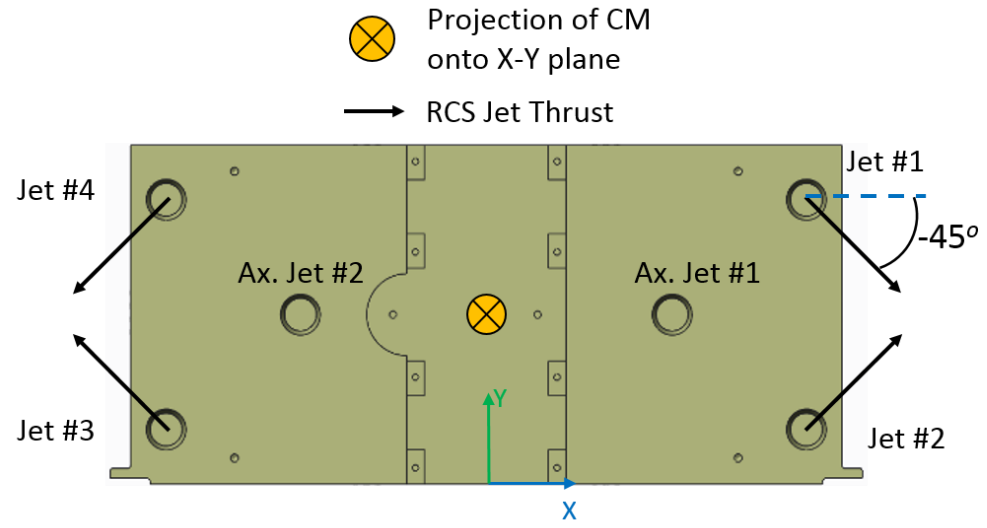

Figure 4. Conceptual image of the RCS unit showing the four attitude control jets and two axial jets. The force directions are visualized as black arrows, and the projection of the spacecraft Center of Mass (CM) is also shown on the RCS unit.

\section{3. $A M T$}

The AMT unit, shown in Figure 5, lies between the avionics bus and the solar sail deployment mechanism. The AMT allows relative translation between the two halves of the spacecraft in the X-Y plane, parallel to the deployed sail plane. This allows the AMT to move the spacecraft's Center of Mass (CM) relative to the solar sail. By doing so, the solar sail thrust can be trimmed to act through the CM, minimizing the solar torque. This is described in greater detail in Section IV-A. Also, details about the AMT mechanical design development can be found in [8].

The AMT is a two degree-of-freedom actuator that uses two lead screws, driven by two stepper motors, that push against each side of the interface to achieve planar motion. The interfacing sides slide relative to one another using carriages with bearings on sliding rails for each translating axis, as shown in Figure 6. A gear system is used for the $\mathrm{Y}$-axis for a more compact volume arrangement of the two stepper motors, increasing range of motion. The AMT achieves a maximum relative translation between the avionics bus and the rest of the spacecraft of $\pm 8 \mathrm{~cm}$ in the $X$ axis and $\pm 3.4 \mathrm{~cm}$ in the $\mathrm{Y}$-axis. The combination of the stepper motor's rotational speed and lead screw pitch gives the AMT translating speeds of $0.3 \mathrm{~mm} / \mathrm{s}$ along the $\mathrm{X}$-axis and $0.1 \mathrm{~mm} / \mathrm{s}$ along the $\mathrm{Y}$-axis. The $\mathrm{Y}$-axis is slower due to the gear reduction.

The AMT has a position accuracy of approximately $0.01 \mathrm{~mm}$. This fine position accuracy is needed to achieve the minimum residual solar torque once the AMT control system has reached a trim position. The lead screws have bearings on the opposite end from the stepper motor and are housed in the aluminum chassis. The stepper motors have tape heaters and temperature sensors to keep them within their operational temperature. Finally, limit switches are placed at one end of each axis to indicate that the maximum travel range has been reached. The limit switches allow for on-orbit reset of AMT position in case of gear or lead screw slippage, ensuring that the AMT operates within its maximum range of motion. 


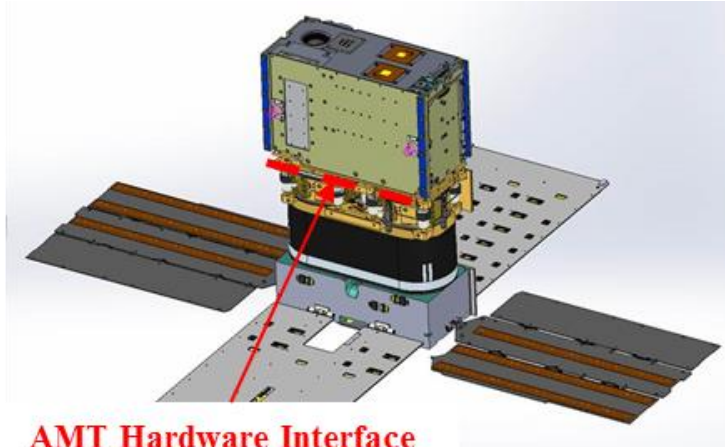

AMT Hardware Interface

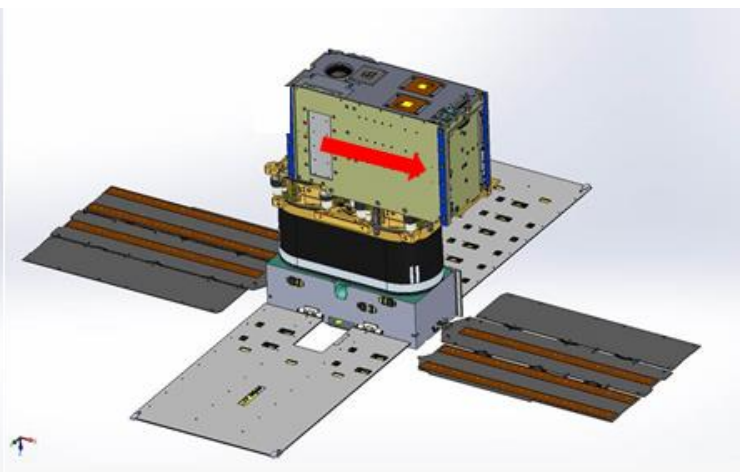

Figure 5. (left) The AMT is located between the spacecraft avionics bus and the sail deployer mechanisms. (right) The AMT displaces the avionics bus relative to the solar sail and RCS unit, shifting the spacecraft's center of mass relative to the center of pressure of the sail.

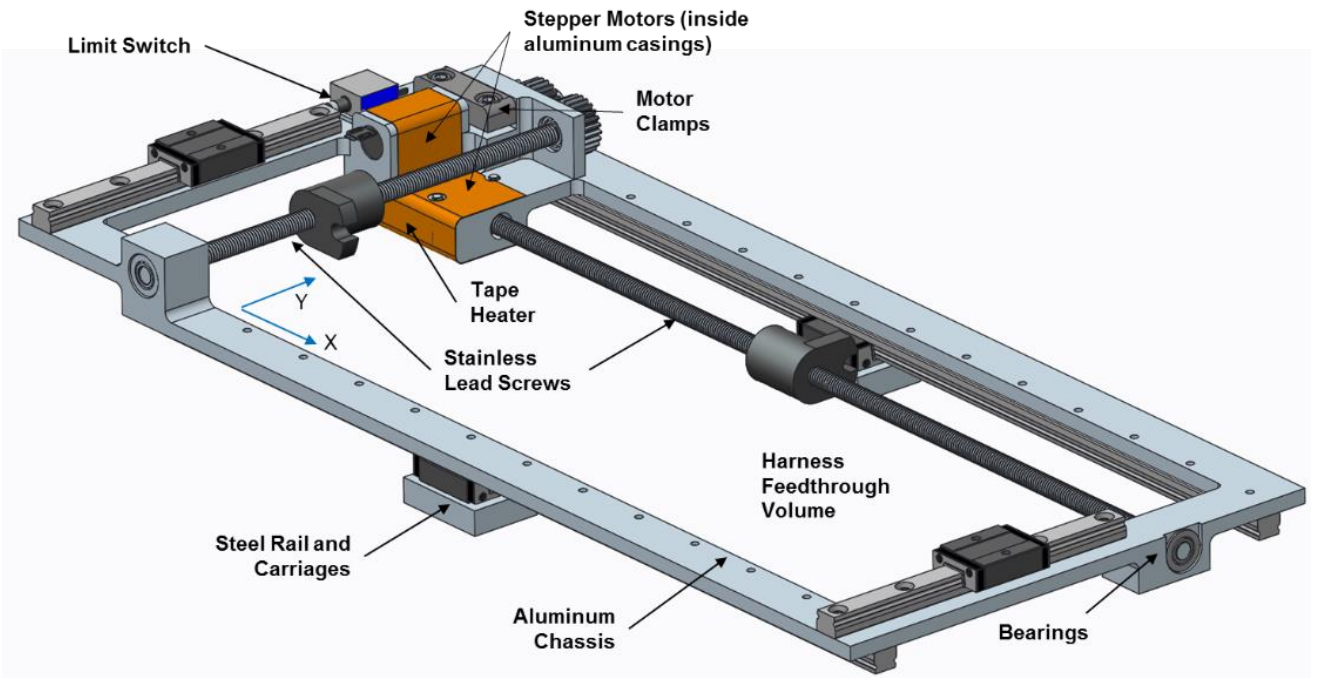

Figure 6. AMT mechanical components, including motors, lead screws, and bearing carriages.

\section{B. Attitude Determination Sensors}

A brief description of the attitude sensor is provided below, but further details may be found in Stiltner et al. [5] and Orphee et. al. [7].

\section{Sun Sensors}

NEA Scout has three sun sensor units from Blue Canyon Technologies. Two sun sensor units are located on the avionics bus, and the third is located on the RCS as shown in Figure 1. Each unit consists of four photodiodes arranged in a pyramid-like orientation. The sun sensors provide a low accuracy attitude solution and are primarily used for initial sun pointing after NEA Scout is ejected. They will also be used during safe mode operations when attitude determination from the star-tracker or IMU are not available.

\section{IMU}

A Sensonor STIM $300^{5}$ Inertial Measurement Unit (IMU) was selected for NEA Scout due to its small form-factor and sufficient noise properties. The IMU gyroscope measurements are used to determine body rates in the attitude control system when star tracker rates are not available. These situations include high spacecraft body rates or if the star tracker sensor saturates due to the Sun, Earth, or Moon being in the field of view.

\section{Star Tracker}

\footnotetext{
${ }^{5}$ http://www.sensonor.com/gyro-products/inertial-measurement-units/stim300.aspx 
The Blue Canyon Technologies (BCT) Thin-Slice Nano Star Tracker (NST) ${ }^{6}$ is the primary attitude and rate sensor onboard NEA Scout. The star tracker provides both inertial attitude and body angular rates. The angular rates are computed by backward differencing of the current and previous attitude quaternions, and are filtered prior to being used by the ACS.

\section{AMT Working Principle, Mission Requirements, and Control Design}

\section{A. AMT Working Principle}

The AMT allows the spacecraft to align its center of mass with the center of pressure of the solar sail, as shown in Figure 7. The sunlight exerts pressure on the sail which can be modeled as a single solar thrust force acting at a point on the sail, called the center of pressure. If the solar sail thrust force is not aligned with the center of mass, it will cause torque on the spacecraft. In that case, the AMT control system will translate the avionics bus in the X-Y plane (parallel to the deployed sail plane) to align the center of mass with the sail center of pressure. This is shown in the trimmed state in Figure 7. Using a two-dimensional translation of the center of mass was explored by NASA's New Millennium Program Space Technology 7 (ST7) [9, 10]. However, ST7 opted for a two-axis gimbaled controlled boom connecting the sail geometric center and the spacecraft bus. Instead, the AMT achieves the same two-dimensional translation using a more compact mechanism that can fit in the available $6 \mathrm{U}$ volume.

At the trimmed state, the net toques on the spacecraft are minimized. However, the above description is an oversimplification to help illustrate the concept. In reality, the sail not only produces normal forces, but it also generates tangential forces to the sail. This is described in more detail by the custom sail force and torque model developed for NEA-Scout by Heaton et al. [611]. The custom sail force and torque model takes into account the deployed sail deformation caused by thermal effects, sail tension, and optical properties of the sail material. The accuracy of the solar sail force and torque models is important in order to design the AMT's translational capacity and position accuracy to meet mission requirements.

The AMT control system was designed to move the spacecraft center of mass to minimize the net resulting torque and keep the torque below a determined threshold. The AMT control system uses feedback from the reaction wheels' speeds to compute a new relative position of the avionics bus needed to trim all external torques and manage reaction wheel momentum. The AMT feedback control system continuously computes a new relative position of the spacecraft avionics box, which will be referred to as the "AMT commanded position" or "AMT position" for short, to maintain the spacecraft environmental torque within requirements. The AMT can trim environmental torque by converging to an $\mathrm{X}$, $\mathrm{Y}$ position, the trim position point, that results in a zero net spacecraft torque in the $\mathrm{X}$ and $\mathrm{Y}$ axes. Second, by purposely moving the AMT away from the trim point, the AMT can produce a favorable torque to manage momentum from the reaction wheels in the $\mathrm{X}$ and $\mathrm{Y}$ axes. The RCS will be used for the $\mathrm{Z}$-axis (sail normal) momentum management. See Section IV for more details.

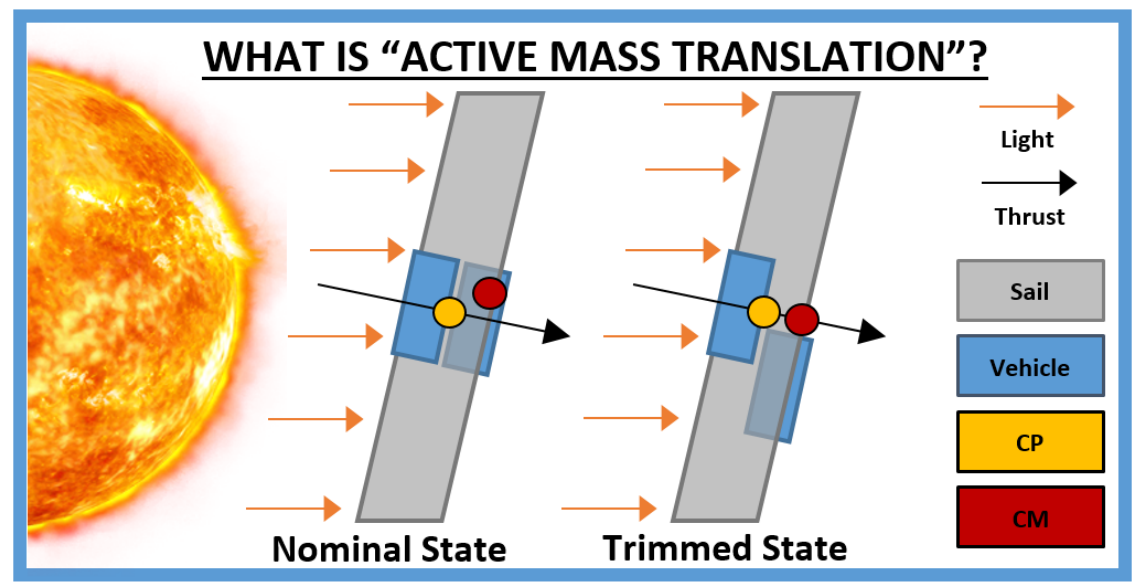

Figure 7. AMT working principle of changing the center of mass to align it with the center of pressure and achieve a trimmed stated.

\footnotetext{
${ }^{6}$ http://bluecanyontech.com/thin-slice-nst/
} 


\section{B. AMT Mission Requirements}

The AMT control system has the following requirements:

1. Shall trim the solar torque in the $\mathrm{X}$ (pitch) and $\mathrm{Y}$ (yaw) axis to 50 nano Newton meters

2. Shall perform momentum management of the reaction wheels in the $\mathrm{X}$ and $\mathrm{Y}$ axes

3. Shall minimize AMT hardware duty cycle and power use

The AMT can only manage torque in the X and Y axis (referred to as pitch and yaw, respectively) and cannot independently control the torque in the sail normal Z-axis (reffered to as roll). The momentum management of the sail normal axis will be done using RCS. However, the solar torque on the sail normal axis is at least one order of magnitude less than the pitch and yaw torques, resulting in low RCS propellant consumption. The RCS is also used as a backup momentum management system for off-nominal situations, such an AMT motor failure. This combination of AMT and RCS for momentum managemen, leverages the capabilities of both system while minimizing RCS propellant consumption and increasing system failure tolerance.

\section{AMT Control Design}

The AMT uses proportional-integral feedback control of the spacecraft body momentum error vector, $\bar{H}_{\text {error }}$ in Eq. (1), to compute an AMT position in the $\mathrm{X}$ and $\mathrm{Y}$ axis. The pre-multiplying matrix in Eq. (1) assigns the correct motion direction according the NEA-Scout coordinate frame for a given momentum error. The momentum error vector, Eq. (2), is defined as the commanded reaction wheel momentum, $\bar{H}_{c m d}$, minus the actual reaction wheel momentum, $\bar{H}_{r w}$. The spacecraft commanded momentum, used to generate a slew maneuver, is the mass moment of inertia matrix of the spacecraft, $\left[I_{s c}\right]$, times the spacecraft commanded body rates vector, $\bar{\omega}_{c m d}$, Eq. (3). The negative sign in Eq. (3) is to reflect that a commanded positive spacecraft momentum causes a negative reaction wheel momentum.

The reaction wheel momentum is simply the summation of the momentum from each reaction wheel in the spacecraft body frame, Eq. (4), where $I_{r w}$ is the reaction wheel moment of inertia along its spin axis, $i$, and $\bar{\omega}_{r w i}$ is the reaction wheel angular velocity vector in the spacecraft body frame of wheel $i$, and $n$ is the number of reaction wheels (four) for NEA-Scout.

$$
\begin{gathered}
{\left[\begin{array}{l}
X_{A M T} \\
Y_{A M T}
\end{array}\right]=\left[\begin{array}{rr}
0 & -1 \\
1 & 0
\end{array}\right]\left[K_{p} \bar{H}_{\text {error }}+K_{i} \int \bar{H}_{\text {error }} d t\right]} \\
\bar{H}_{\text {error }}=\bar{H}_{c m d}-\bar{H}_{r w} \\
\bar{H}_{c m d}=-\left[I_{s c}\right] \bar{\omega}_{c m d} \\
\bar{H}_{r w}=\sum_{i=1}^{n} I_{r w i} \bar{\omega}_{r w i}
\end{gathered}
$$

This control law guarantees minimum momentum error during attitude holds or slew maneuvers. In this manner, the AMT control system commands different AMT positions during slews that minimize momentum error along the slew path, keeping the spacecraft close to a trim state during the slew maneuver. Momentum management during slew maneuvers is essential for NEA Scout due to its large moment of inertia, resulting in in small achievable slew speeds, $0.04 \mathrm{deg} / \mathrm{s}$ in pitch or yaw. Therefore, momentum accumulation during long slews would have exceeded reaction wheel capacity.

The AMT position control uses deadbands to avoiding excessive duty cycling. To trigger an AMT position command, an absolute reaction wheel momentum value $\bar{H}_{r w}>\bar{H}_{r w_{-} \text {deadband }}$, must be exceeded. Each axis, X and Y, can be commanded independently if the deadband has been exceeded for that axis. However, to shut-off the AMT commands, the reaction wheel momentum and spacecraft torque have to fall within specified deadbands as shown in Eq. (5) .

$$
\bar{H}_{r w}<\bar{H}_{r w_{-} d e a d b a n d} \text { and } \dot{\bar{H}}_{r w}<\dot{\bar{H}}_{r w_{-} \text {deadband }}
$$


In Eq. (5), $\dot{\bar{H}}_{r w}$ is the time derivative of the reaction wheel momentum vector (a body torque) that guarantees the torque has reached the deadband before turning the controller off. Finally, to further minimize duty cycle, the AMT controller sends a new position command update to the hardware once every 100 seconds.

\section{AMT Control Performance}

\section{A. Attitude Hold}

After solar sail deployment, NEA-Scout will perform attitude hold using reaction wheels, and the AMT controller will be enabled. Initially, a sun pointing attitude will be held. But soon after, a sequence of higher Sun Incidence Angles (SIA) will be exercised to characterize the actual on-orbit solar torques caused by the deployed sail. For NEAScout, the largest solar torque about the spacecraft center of mass, approximately $2.5 \mathrm{mN}$-m (milli-Newton meters), and is expected to occur approximately at 45 degrees SIA. NEA-Scout is expected to operate within approximately 50 degrees SIA for most of its mission. However, near the asteroid approach phase, a maximum SIA of up to 70 degrees may be required to achieve Earth communications.

A 45-degree SIA attitude hold state was simulated and results are shown in Figure 8. With the AMT staring at its stowed position, Figure 8 (left) shows the solar torque as the AMT is autonomously commanded to new positions until the solar torque is less than the required maximum of $50 \mathrm{nN}-\mathrm{m}$ (nano-Newton meters), achieved after approximately 5000 seconds as shown in Figure 8 (right).
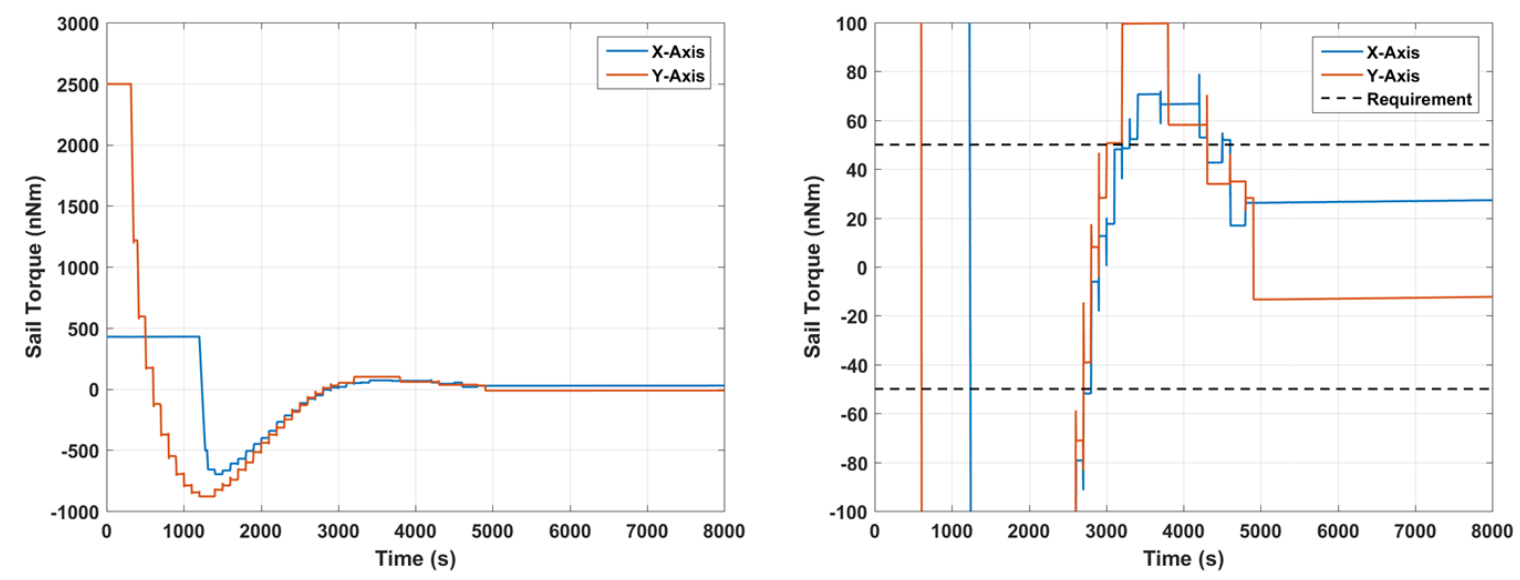

Figure 8. (left) Solar torque on the spacecraft at a sun incidence angle of $\mathbf{4 5}$ degrees while the AMT moves to a trim position. (right) Shows the zoomed-in solar torque achieving less than $50 \mathrm{nN}-\mathrm{m}$ requirement after 5000 seconds, approximately.

Figure 9 (left), shows the reaction wheel response to the solar torque for a $45 \mathrm{deg}$. SIA attitude hold, and the AMT momentum management. The AMT autonomously positions itself to create a counter torque, Figure 9 (right) to reduce the reaction wheel momentum. The AMT position initially overshoots the equilibrium position to cause the counter acting torque needed for reaction wheel desaturation. Once the reaction wheel momentum is reduced below the momentum deadband $\left(5 \times 10^{-5} \mathrm{Nms}\right)$, the AMT controller commands a position that minimizes the solar torque within the required $50 \mathrm{nN}-\mathrm{m}$ bounds and shuts-off after approximately 5000 seconds. 

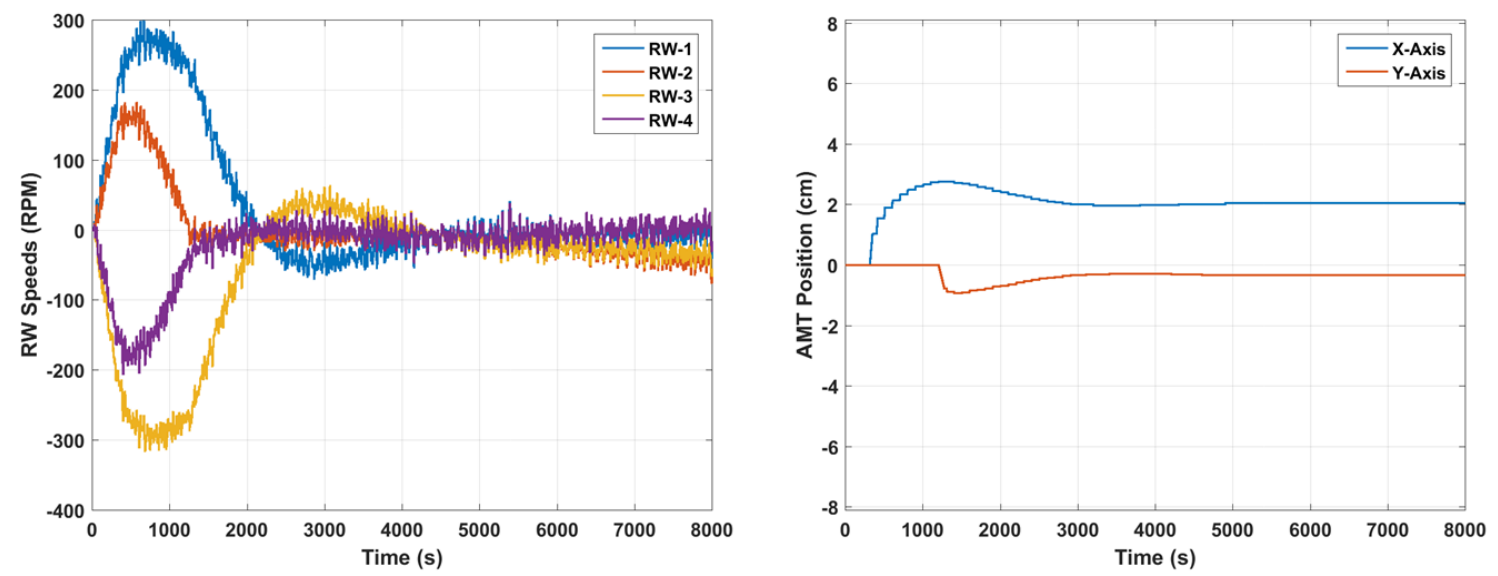

Figure 9. (left) Reaction wheel response to solar torque for 45 degree SIA attitude hold and active AMT control system. (right) AMT position response to perform reaction wheel desaturation and achieve a trimmed position.

\section{B. Slew Maneuvers}

Duringslew maneuvers, the reaction wheel momentum will be commanded to increase or decrease in order to induce an equal and opposite momentum on the spacecraft. However, the AMT controller responds to the momentum error (commanded minus the actual reaction wheel momentum) as described in Section IV-C. This allows the AMT to keep the spacecraft close to a trim condition during long duration slews, avoiding reaction wheel saturation. A 0 to 70 degrees SIA slew was simulated to evaluate the AMT performance under the largest required SIA during the mission. Figure 10 (left) shows the attitude error versus time, and Figure 10 (right) shows the commanded and actual spacecraft body rates versus time during the single axis slew maneuver.
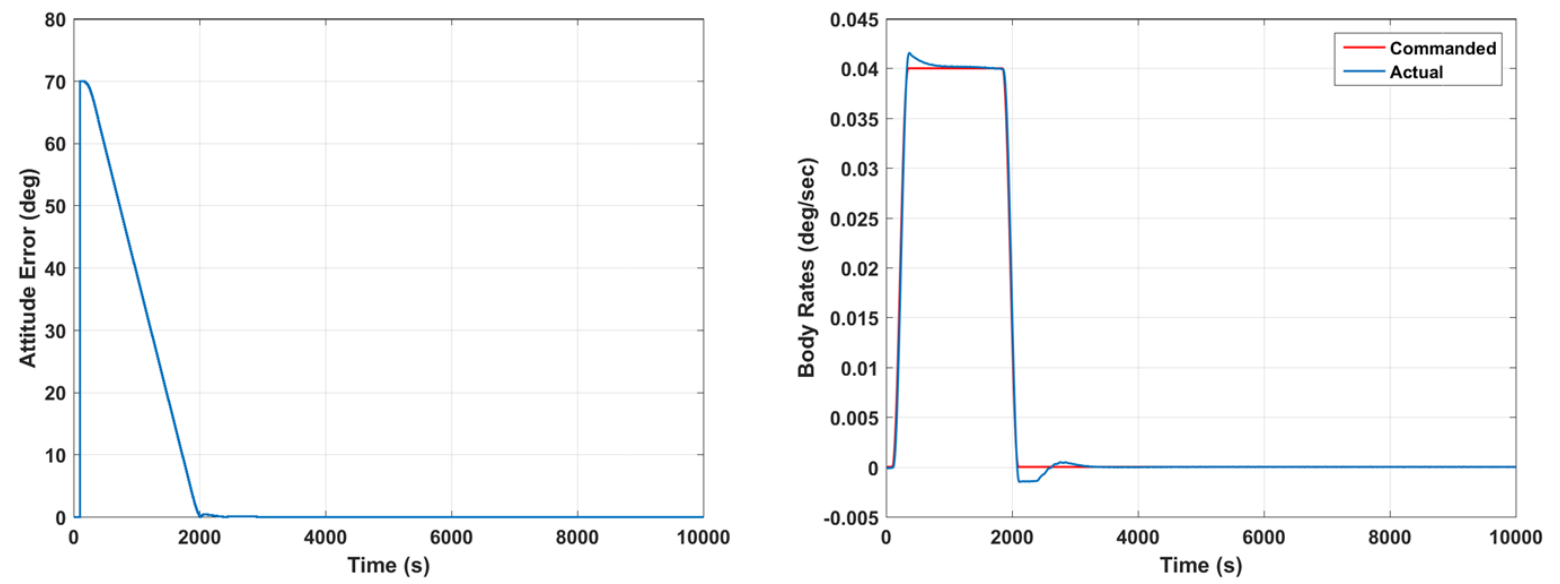

Figure 10. (left) Attitude error during slew. (right) Commanded and actual spacecraft body rates during the slew.

The slew profile shown in Figure 10 (right) is used to maximize the time spent at the maximum slew rate of 0.04 degrees per second in the solar sail deployed configuration. The solar torque during the slew maneuver is shown in Figure 11. At the end of the slew maneuver, a large transient is caused by the AMT's lagged response. The transient settles to the required $50 \mathrm{nN}-\mathrm{m}$ after approximately 5000 seconds. 

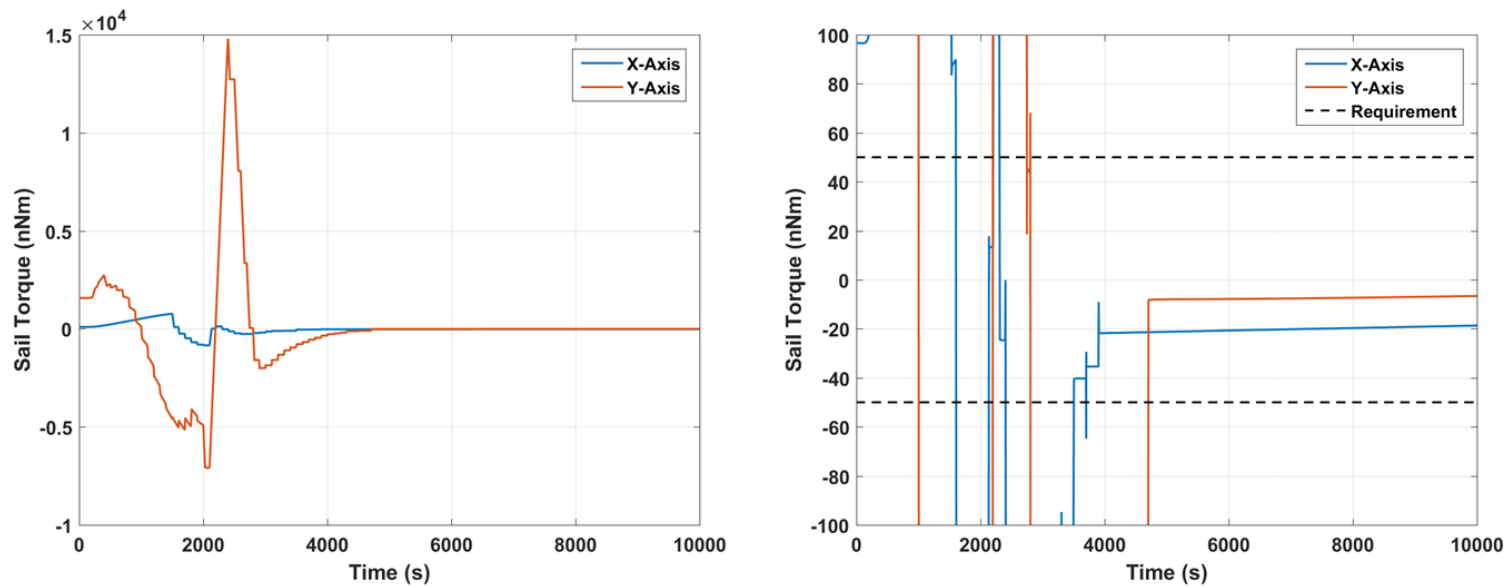

Figure 11. (left) Solar torque on the spacecraft during a 70 deg. angle while the AMT moves to a trim position. (right) Shows the zoomed-in solar torque achieving less than $50 \mathrm{nN}$-m requirement after 5000 seconds, approximately.

The reaction wheel response and AMT positions are shown in Figure 12. The AMT position control causes the reaction wheel momentum not to grow significantly once the maximum slew rate was achieved, following the desired slew rate profile. Avoiding momentum growth during slews reduces the risk of reaction wheel saturation. Once the AMT reaches equilibrium, the AMT is commanded to shut-off for energy and hardware duty-cycle reduction.
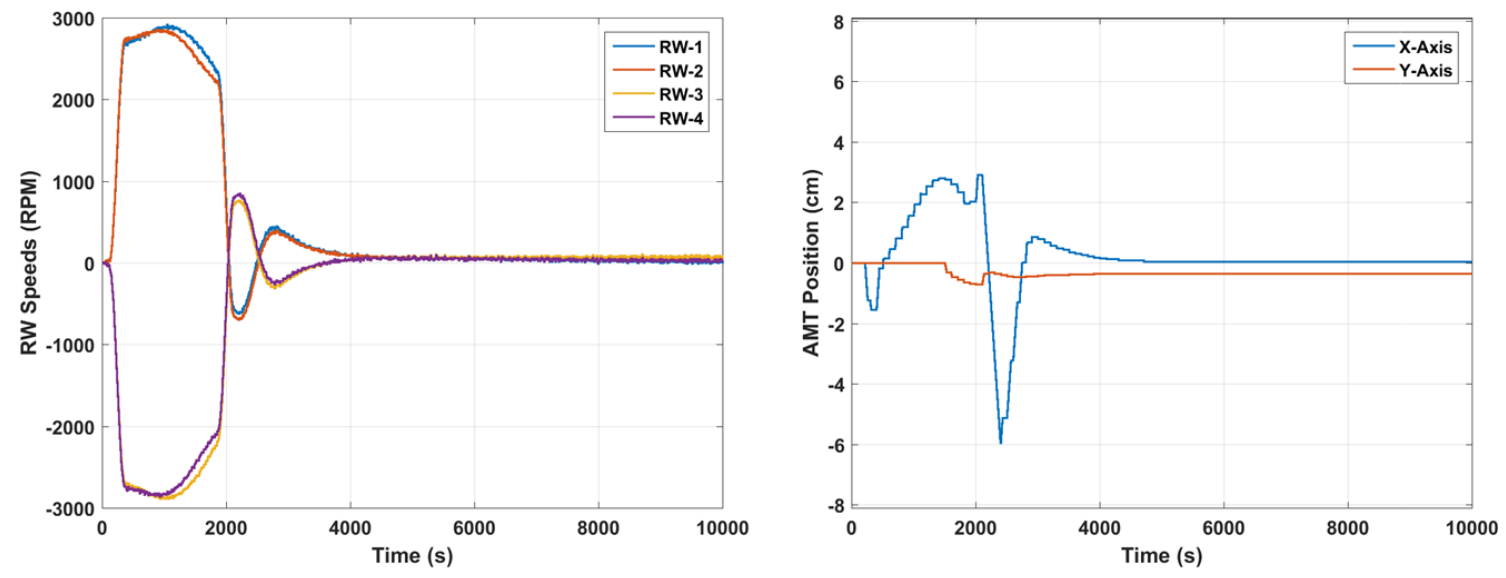

Figure 12. (left) Reaction wheel response to solar torque for 45 degree SIA attitude hold and active AMT control system. (Right) AMT position response to perform reaction wheel desaturation and achieve a trimmed position.

\section{Conclusions}

This paper provides an overview of the NEA Scout mission and attitude control system with particular emphasis on the novel Active Mass Translator (AMT) hardware and its control logic. The AMT is a propellantless, compact, and autonomous solar torque management system. The AMT control system autonomously shifts the center of mass of the spacecraft to trim the dominant solar torques about the spacecraft's $\mathrm{X}$ and Y axes. Traditional solar torque management systems using a reaction control system would have required much more propellant, increasing the total mass and volume of the spacecraft. An increase in spacecraft mass and volume not only means higher launch costs, but also means a reduction of in-space acceleration, increasing the time-of-flight. For deep-space solar-sail missions using non-radiation tolerant avionics, a reduced time-of-fight allows a wider range of possible targets within the electronics' radiation lifetime. The baseline NEA Scout mission will take approximately two and a half years. The use of the AMT allows for less carried propellant and smaller capacity reaction wheels, enabling a $6 \mathrm{U}$ spacecraft volume and increased range of possible missions. 


\section{References}

1 NASA JPL Small-Body Database Browser, 1991 VG. Link: https://ssd.jpl.nasa.gov/sbdb.cgi?sstr=1991VG.

2 McNutt, L., "Near-Earth Asteroid (NEA) Scout", AIAA SPACE 2014 Conference and Exposition, AIAA SPACE Forum, (AIAA 2014-4435)

3 Vinh, B., "Near Earth Asteroid (NEA) Scout Solar Sail Implementation," Annual AIAA/USU Conference on Small Satellites; 30th; 6-11 Aug. 2016; Logan, UT; United States, 2016, available at: https://ntrs.nasa.gov/search.jsp?R=20160011083.

4 Johnson, L., "Near Earth Asteroid (NEA) Scout", The Fourth International Symposium on Solar Sailing, 17002, ISSS, Kyoto, Japan, 2017. Available at http://www.jsforum.or.jp/ISSS2017/papers/index.html.

5 Stiltner, B., "Cold Gas Reaction Control System for the Near Earth Asteroid Scout CubeSat", AIAA SPACE and Astronautics Forum and Exposition, AIAA SPACE Forum, (AIAA 2017-5185)

6 Heaton, A., "Near Earth Asteroid Scout Solar Sail Thrust and Torque Model", The Fourth International Symposium on Solar Sailing, 17055, ISSS, Kyoto, Japan, $2017 . \quad$ Available at http://www.jsforum.or.jp/ISSS2017/papers/index.html.

7 Orphee, J., "Solar Sail Attitude Control System for the NASA Near Earth Asteroid Scout Mission", The Fourth International Symposium on Solar Sailing, 17091, ISSS, Kyoto, Japan, $2017 . \quad$ Available at http://www.jsforum.or.jp/ISSS2017/papers/index.html.

8 Few, A. "Development of a High Performance, Low Profile Translation Table with Wire Feedthrough for a Deep Space CubeSat", 43rd Aerospace Mechanisms Symposium, Santa Clara, CA, USA, 2016. Available at: https://ntrs.nasa.gov/search.jsp?print=yes\&R=20160007074

9 Price, H., "Design for a Solar-Sail Demonstration Mission," Space Technology and Applications International Forum (STAIF 2000), Feb. 2001, available at: https://trs.jpl.nasa.gov/handle/2014/12303?show=full

10 Murphy, D. M., "Scalable Solar-Sail Subsystem Design Concept," Journal of Spacecraft and Rockets, Vol. 40, No 4, 2003, pp. 539-547. 